Documentation et bibliothèques

\title{
Le service de repérage de l'information sur disque optique à l'École Polytechnique de Montréal
}

\section{Richard Dumont et Luc Foucault}

Volume 38, numéro 4, octobre-décembre 1992

URI : https://id.erudit.org/iderudit/1028768ar

DOI : https://doi.org/10.7202/1028768ar

Aller au sommaire du numéro

\section{Éditeur(s)}

Association pour l'avancement des sciences et des techniques de la documentation (ASTED)

\section{ISSN}

0315-2340 (imprimé)

2291-8949 (numérique)

Découvrir la revue

Citer cet article

Dumont, R. \& Foucault, L. (1992). Le service de repérage de l'information sur disque optique à l'École Polytechnique de Montréal. Documentation et bibliothèques, 38(4), 197-202. https://doi.org/10.7202/1028768ar

Tous droits réservés (C) Association pour l'avancement des sciences et des techniques de la documentation (ASTED), 1992
Ce document est protégé par la loi sur le droit d'auteur. L'utilisation des services d'Érudit (y compris la reproduction) est assujettie à sa politique d'utilisation que vous pouvez consulter en ligne.

https://apropos.erudit.org/fr/usagers/politique-dutilisation/ 


\title{
Le service de repérage de l'information sur disque optique à l'École Polytechnique de Montréal
}

\author{
Richard Dumont \\ responsable du Service de repérage de l'information \\ sur disque optique (SRIDO) et du service de téléréférence \\ École Polytechnique de Montréal \\ Luc Foucault \\ bibliothécaire de référence \\ responsable de la formation documentaire \\ École Polytechnique de Montréal
}

\section{L'École Polytechnique de Montréal}

La communauté polytechnicienne se compose essentiellement de 5000 étudiants, 210 professeurs et 175 chercheurs, ce qui fait de l'École Polytechnique de Montréal la plus grande école de formation d'ingénieurs au Canada. Elle dispense des cours regroupés dans les secteurs suivants: génie biomédical, génie chimique, génie civil, génie électrique, génie énergétique, génie géologique, génie industriel, génie informatique, génie minier, génie des matériaux, génie mécanique, génie physique et mathématiques appliquées.

\section{La bibliothèque}

Outre la clientèle universitaire traditionnelle (étudiants, professeurs, chercheurs), la bibliothèque dessert les clientèles extérieures telles qu'ingénieurs et industrie/s parl'intermédiaire du Service d'information documentaire de l'École Polytechnique (SIDEP). Créé en 1985, le SIDEP est un organisme à but non lucratif qui opère sur une base de recouvrement des frais encourus.

La collection de la bibliothèque comprend environ 120000 monographies et rapports techniques, 950 abonnements à des périodiques et 30000 normes techniques. Des catalogues industrie/s, des logiciels, des documents audiovisuels et des cartes complètent la collection.

Les banques de données sur disque optique existent depuis quelques années déjà et constituent des outils appréciables dans l'arsenal du chercheur. Bien que la mise en place d'un service de repérage de l'information sur disque optique (SRIDO) requiert d'importantes ressources humaines et matérielles, les bibliothèques universitaires se doivent d'offrir un tel service. C'est dans ce contexte que le SRIDO a été instauré à la bibliothèque de Polytechnique. Cet article en présente l'implantation en insistant plus particulièrement sur la formation documentaire. Les choix d'équipements et de banques de données, la promotion effectuée, les statistiques d'utilisation, les impacts sur les autres services ainsi que les développements prévus sont également discutés.

\section{Contexte initial}

Depuis le milieu des années 80 , la bibliothèque s'intéressait aux banques de données sur disque optique et les développements étaient suivis avec attention. Au printemps 1989, la décision de mettre sur pied le SRIDO était arrêtée.

Nous devions d'abord choisir les banques de données qui répondaient aux besoins documentaires les plus pressants et dont l'utilisation anticipée justifierait les coûts d'abonnement. Quatre banques remplissaient ces critères: Compendex Plus, Applied Science \& Technology, Books in Print Plus et Electre-Biblio (Livres disponibles). Alors que les deux premières visaient à satisfaire les besoins documentaires de notre clientèle, les deux dernières allaient devenir principalement un outil de travail pour le personnel du service des acquisitions de la bibliothèque.
Notre choix d'équipements fut surtout dicté par le désir de limiter au minimum les interventions des usagers avec les lecteurs de disque optique, l'imprimante ou les commandes du DOS. Or, à elle seule, la banque de données Compendex Plus posait certaines contraintes matérielles. En effet, cette banque répartie sur cinq disques rendait souhaitable l'acquisition de plusieurs lecteurs afin de réduire les manipulations de disques par les usagers. Cette configuration permet aussi d'instaurer une interface de type «menu» pour gérer l'appel des banques de données, évitant ainsi l'utilisation des commandes du DOS. De plus, ces appareils éliminent l'entreposage des disques non utilisés et conséquemment leur circulation. Nous avons donc opté pour trois lecteurs multiples (tour à quatre lecteurs) fabriqués par la compagnie ONLINE. Dans le même esprit, nous avons choisi une imprimante laser de marque LaserJet III d'Hewlett Packard. En plus d'être silencieuse et rapide, elle réduit la manipulation de papier et permet de récupérer une partie des coûts d'impression en y adjoignant un contrôleur de copies (carte de débit). Finalement, deux micro-ordinateurs (IBM $P S / 2$ ) sont venus compléter notre sélection d'équipements.

Pour financer l'achat des équipements et les abonnements d'une année aux banques, la bibliothèque a présenté une demande d'assistance financière de $36500 \$$ à la Fondation de Polytechnique ${ }^{1}$. La

1. La Fondation de Polytechnique est un instrument de levées de fonds. Les sommes recueillies sont par la suite distribuées sous forme de bourses à des étudiants, de subventions à la recherche ou d'investissements (équipements, biens immobiliers). 
demande fut acceptée intégralement. Les coûts d'abonnements aux banques de données pour les années subséquentes seraient défrayés par le budget régulier de la Bibliothèque.

\section{Instauration}

L'instauration du SRIDO s'est effectuée graduellement (tableau 1). Cela nous a permis d'implanter adéquatement Compendex Plus, de nous assurer de la compatibilité et de la performance des équipements et de nous familiariser avec les logiciels d'interrogation. Le tableau 2 présente les principales difficultés techniques que nous avons rencontrées. Parallèlement, des politiques de fonctionnement ont été élaborées (tableau 3), de la documentation d'accompagnement a été préparée et des activités de formation ont été mises sur pied.

En terminant, mentionnons que les coûts d'abonnement à certaines banques ont été défrayés par d'autres départements de l'École: CCinfo (santé et sécurité au travail), Sigma-aldrich (fiches toxicologiques) et Hydat (données hydrologiques canadiennes). À cet égard, un protocole d'entente pour le dépôt de documents sur disque optique à la Bibliothèque a été développé.

\section{Promotion}

Outre les articles parus dans les journaux étudiants, trois activités de promotion majeures ont eu lieu. Avant même le lancement du service, un stand de démonstration a été tenu au café étudiant.
Cette activité de quelques heures a permis de constater que cette technologie suscitait de l'intérêt chez les étudiants. Plusieurs nous ont questionnés dans les semaines qui suivirent parfois sans même être allés au stand. Ensuite, il y a eu l'inauguration officielle du service auprès de la Direction de l'École. C'était pour nous l'occasion de remercier publiquement la Fondation de Polytechnique pour son support financier. Enfin, la dernière activité visait plus particulièrement les professeurs et les chercheurs de l'Ecole. Pour l'occasion, nous avions déplacé les équipements du SRIDO au Salon des professeurs pour une journée entière. Deux bibliothécaires étaient sur place pour répondre aux questions et effectuer des démonstrations. Les professeurs avaient tous reçu préalablement un dossier

\section{TABLEAU 1}

\section{CHRONOLOGIE DES PRINCIPAUX ÉVÉNEMENTS}

printemps 1989

janvier 1990

février 1990

mai 1990

juin 1990

août 1990

octobre 1990

novembre 1990

janvier 1991

janvier 1992

décembre 1992
Le premier micro-ordinateur est acquis.

Demande d'assistance financière adressée à la Fondation de Polytechnique.

Réponse positive de la Fondation de Polytechnique; les banques de données Compendex Plus, Books in Print Plus et Electre-Biblio sont commandées de même qu'un multilecteur. Stand de démonstration au café étudiant.

Familiarisation avec les banques de données et les équipements; élaboration d'un manuel de politiques et procédures; préparation de documentation d'accompagnement et conception d'activités de formation.

Premier poste de travail disponible. À compter du 18 juin, le service est accessible aux usagers.

Mise en service du deuxième poste de travail. Acquisition de l'imprimante laser et installation du contrôleur de copies. Ajout des banques Sigma-aldrich et CCinfo.

Inauguration officielle du SRIDO.

Activité de promotion auprès des professeurs.

Ajout des banques Applied Science \& Technology et Hydat. Élaboration d'un didacticiel pour Applied Science \& Technology.

Ajout de la banque Inspec.

Ajout d'un troisième poste de consultation et des banques Automated patent searching et Move. 
comprenant l'invitation, le dépliant du service et des fiches descriptives des banques de données.

\section{Formation documentaire}

Afin de présenter un tableau complet de notre programme de formation docu- mentaire pour les banques de données sur disque optique, nous dresserons d'abord un portrait de nos usagers. Deux dénominateurs communs les caractérisent. Premièrement, la plupart possèdent une culture informatique, ceci dû essentiellement à leur formation académique. Deuxièmement, peu d'usagers ont une formation documentaire adéquate. A priori, la maîtrise de l'informatique constitue un apport positif. En effet, la connaissance de l'équipement informatique et la facilité relative à utiliser des logiciels, et conséquemment un logiciel d'interrogation d'une banque de données, contribuent à l'allègement de la formation. Toutefois, la combinaison de

\section{TABLEAU 2}

\section{DIFFICULTÉS TECHNIQUES RENCONTRÉES}

\section{LE PROBLÈME}

Incompatibilité entre les logiciels. Par exemple, il est impossible d'interroger Electre-Biblio immédiatement après avoir utilisé Books in Print Plus. Ce problème est principalement causé par une mauvaise gestion de la mémoire vive de la part des logiciels d'interrogation.

Contrôler l'accès aux disques optiques.

Perte du module mémoire d'un micro-ordinateur. L'électricité statique est probablement la cause de cette défaillance. L'utilisation de chaises de plastique et le tapis du plancher de la bibliothèque sont des facteurs suffisants pour causer ce genre de problème.

Confusion lors de l'impression des résultats. Une imprimante laser imprime une feuille uniquement lorsque la page est pleine ou dès qu'un code de saut de page lui est envoyé. Lorsque la dernière notice demandée par un usager ne remplit pas la page, cette notice demeure dans la mémoire de l'imprimante. Cette situation laissait souvent les utilisateurs perplexes.

Utilisation démesurée de l'imprimante.

Un interrupteur permet de partager l'imprimante entre les deux postes de consultation. Les usagers oublient souvent de placer correctement cet interrupteur et un message d'erreur, souvent très confus, est alors émis par les logiciels d'interrogation.

Contrôler l'accès au système d'exploitation. Bien que les déplacements d'une banque à l'autre soient gérés par une interface-menu, les usagers ont accès assez facilement au DOS. Un usager a même installé sur un poste son propre logiciel de traitement de texte!

Disque rigide effacé au complet par un reformatage de bas niveau (ce type d'opération ne peut être effectué à l'aide des commandes du DOS). Il est le résultat d'un usager assez futé et mesquin ou l'oeuvre d'un virus informatique.

\section{NOTRE APPROCHE}

Il faut réinitialiser le micro-ordinateur avant chaque utilisation des logiciels problématiques. Pour ce faire, nous utilisons un programme utilitaire appelé WARMBOOT.COM diffusé par PC MAGAZINE. Cet utilitaire est inséré dans le programme en lot qui appelle le logiciel d'interrogation.

Nous avions d'abord placé les multilecteurs dans un boîtier verrouillé à clef. Ce dernier possédait de nombreuses ouvertures mais nous avons eu quelques problèmes de surchauffe. Les multilecteurs sont maintenant ancrés à la table avec un câble d'acier et une plaque de plexiglass est placée devant les lecteurs afin d'empêcher le retrait des disques.

Les chaises de plastique ont été remplacées par des chaises de tissu et des tapis antistatiques ont été installés.

Nous avons d'abord entrepris d'éduquer les usagers sur la manière de faire imprimer le contenu de la mémoire de l'imprimante. Sans être complexe, cette opération exige quand même d'appuyer sur trois touches du panneau de contrôle de l'imprimante. Nous avons alors choisi de développer un utilitaire qui envoie un code de saut de page à la fin du fichier d'impression : la demière page est alors imprimée. Cet utilitaire, entièrement transparent à l'usager, a été conçu par un membre du personnel des systèmes informatisés de la bibliothèque.

Nous avons placé un contrôleur de copies sur l'imprimante laser. Ce contrôleur fonctionne à l'aide d'une carte magnétique qui est utilisée un peu partout dans l'École pour les photocopieurs et les machines distributrices. Le coût d'impression, fixé à $0.04 \$$ la page, agit comme ticket modérateur et nous permet de récupérer les frais encourus pour le papier et l'encre.

Encore une fois, c'est notre service des systèmes informatisés qui a fourni la solution. Un utilitaire est activé dès qu'une impression est lancée. Cet utilitaire vérifie si l'imprimante est en état de recevoir le fichier à imprimer. Dans le cas contraire, un message demandant à l'usager de vérifier certains paramètres est affiché à l'écran.

Ce problème n'est pas encore réglé. Bien que l'équipe de référence exerce une surveillance accrue depuis cet incident, aucune disposition sérieuse n'a été mise en place.

Une copie de sauvegarde nous a permis de restaurer le disque rigide. Un programme spécialisé dans la détection de virus informatiques a été installé sur les postes de consultation. 
ces deux éléments, soit une forte culture informatique jumelée à une faible culture documentaire, peut défavoriser un usager. Se sentant relativement en confiance avec l'outil, l'usager s'assoit spontanément devant le poste de travail pour interroger une banque. Puisqu'il réussit à naviguer dans les menus et sous-menus et à obtenir des résultats, l'usager est non seulement persuadé d'avoir bien fait sa recherche mais il est souvent convaincu d'avoir su exploiter correctement les possibilités de recherche. II repartira plus ou moins satisfait selon les informations obtenues, et même qu'advenant de piètres résultats, il remettra rarement en cause son inexpérience.
Sans en être conscients, nos usagers doivent non seulement posséder des connaissances liées à la micro-informatique (équipements, termes ou symboles) et à leur spécialité, mais aussi posséder des notions de base en bibliothéconomie pour effectuer correctement l'interrogation d'une banque de données. Plus précisément, ces connaissances comprennent les concepts d'indexation, de stratégies de recherche, etc. et certains termes tels que notices, descripteurs, etc. souvent rencontrés dans le monde documentaire. Un programme de formation doit donc permettre aux usagers d'acquérir les connaissances documentaires et informatiques; les connaissances liées à la spécialité demeurant sous

\section{TABLEAU 3}

\section{PRINCIPALES MODALITÉS D'UTILISATION DU SRIDO}

Objet du service

Clientèles visées

Formation

Localisation

Heures de disponibilité

Privilège de réservation

Tarification
Permettre aux usagers d'interroger eux-mêmes les banques de données sur disque optique de façon efficace et optimale.

Le SRIDO est offert tant aux clientèles internes qu'externes de la bibliothèque tout comme les autres ressources documentaires.

L'assistance à une séance de formation est facultative. Le mode de formation varie selon les banques de données.

Le SRIDO se situe près des bureaux du service de référence. Cela permet d'assister rapidement l'usager.

Le SRIDO est disponible durant les heures d'ouverture de la bibliothèque. Par ailleurs, seule une assistance matérielle est offerte en dehors des heures de référence.

Afin de favoriser l'utilisation du SRIDO par la clientèle interne et de réduire les attentes longues et imprévisibles, une politique de réservation est en vigueur. Ainsi, un usager a le privilège de réserver une ou deux séance(s) de 30 minutes par jour. Un cahier de réservation est disponible à cette fin. Bien entendu, un usager peut interroger sur le champ un poste de consultation si personne ne l'utilise.

Les banques de données sur disque optique constituent des ouvrages de référence au même titre que les index de périodiques et c'est dans cet esprit que le SRIDO est offert gratuitement. leur responsabilité. C'est pourquoi, notre programme de formation tient compte des quatre aspects suivants : 1) les procédures du service; 2) l'équipement informatique; 3) le logiciel d'interrogation de la banque; 4) la couverture, la structure et l'indexation de la banque.

Le programme doit également prendre en considération le contexte linguistique dans lequel nous sommes: nos usagers sont majoritairement francophones alors que le contenu et le logiciel d'interrogation des banques de données sont généralement en anglais. Cette situation pose certains problèmes spécifiques tant au niveau des utilisateurs que de la bibliothèque.

Chez les usagers, les problèmes les plus importants relèvent des connaissances limitées qu'ils ont de l'anglais. En plus de devoir connaître la traduction anglaise des termes de leur domaine, les usagers doivent maîtriser un minimum de vocabulaire. Par exemple, le terme Phrase ne signifie pas «phrase» mais bien «expression». La distinction entre les termes Entries et Records peut aussi être une source de confusion. Même la signification de Display, de Remove ou encore de When Done cause certaines difficultés d'interprétation.

Pour la bibliothèque, le problème principal qu'engendre l'exploitation d'une banque de données autre que de langue française est l'impossibilité d'utiliser directement la documentation fournie par le producteur. Aussi, la rédaction d'une documentation adéquate est donc souhaitable.

Notre programme de formation documentaire a donc été élaboré en considérant cet ensemble de facteurs. Ainsi, l'insistance a surtout été placée sur les connaissances bibliothéconomiques puisque c'est dans ce secteur que nos usagers montrent le plus de carences. L'informatique est également couverte mais d'une manièremarginale. De plus, le programme tient compte du contexte particulier occasionné par l'utilisation de l'anglais.

Nous avons décidé de laisserla formation facultative. Plusieurs raisons motivaient ce choix. D'abord, nous considérons que notre rôle est de favoriser, non pas d'obliger, une utilisation adéquate des 
ressources. Deuxièmement, nous ne désirions pas instaurer des mesures visant à contrôler la présence des usagers à la formation. Finalement, l'interrogation des banques s'effectue généralement de manière conviviale.

L'approche choisie entre les divers modes de formation a été faite en fonction des banques de données et de la fréquence d'utilisation anticipée. Avec Compendex Plus et Inspec, nous avons adopté une «pédagogie traditionnelle», c'est-à-dire un guide de l'usager jumelé à une séance de formation facultative. En plus de faciliter l'apprentissage, cela permet à l'usager d'acquérir plus facilement les termes liés à la documentation. De juin 90 à septembre 92,163 séances ont été données à 333 personnes, ce qui représente une moyennemensuelle de six séances à raison de deux personnes par séance.

Avec la banque $A S T$, nous avons privilégié une formation à l'aide d'un didactitiel. En plus de permettre à l'usager de pro- gresser à son propre rythme, le didacticiel assure une formation constante contrairement aux séances de formation données verbalement. Deux raisons ont motivé ce choix. Premièrement, la structure de la banque AST se prêtait bien à l'utilisation d'un didacticiel. En effet, l'absence de résumé, l'indexation limitée et la structure du logiciel d'interrogation favorisent cette approche. Deuxièmement, l'équipe de référence pouvait difficilement offrir des séances de formation sur une autre banque compte tenu de ses effectifs. Les statistiques d'utilisation du didacticiel indiquent qu'une vingtaine d'usagers le consultentmensuellement et y consacrent 7 minutes en moyenne.

Dans le cas des autres banques, nous avons jugé qu'il était préférable de donner uniquement des séances de formation sur demande. Nous estimons que leur utilisation est trop restreinte pour justifier l'investissement considérable de ressources qu'exigent l'élaboration de didacticiels ou des cours de formation. Du matériel di-

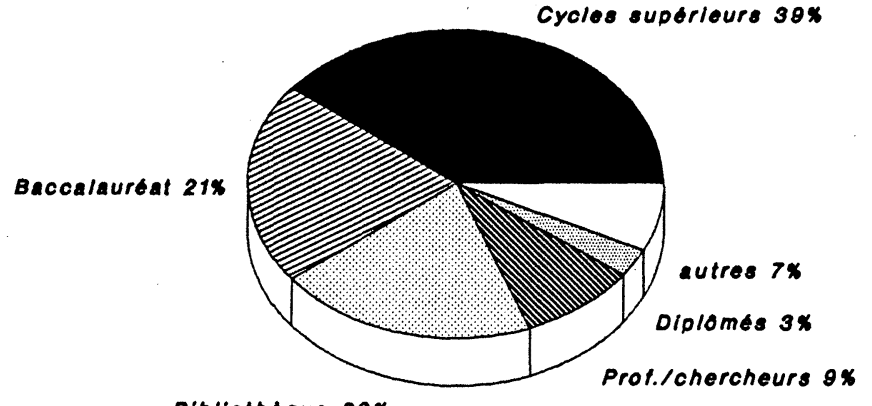

Bibliotheque 20\%

flgure 1: Statut des usagers du SRIDO

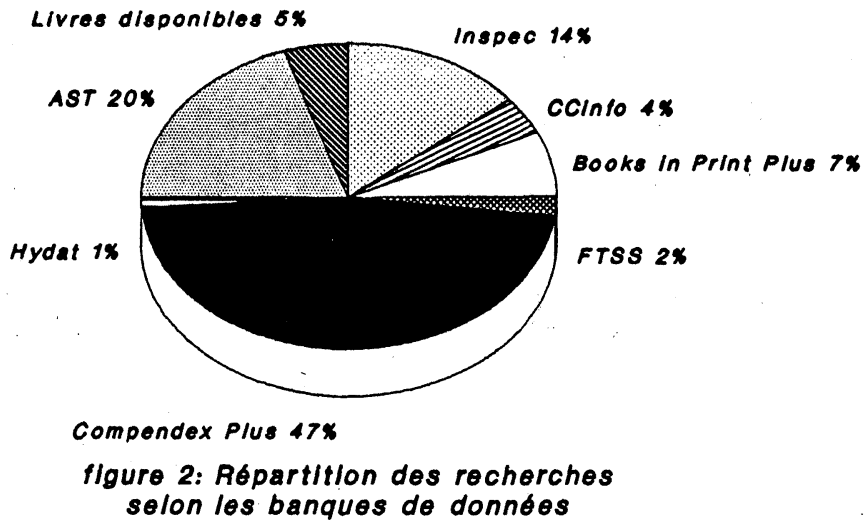

dactique sous forme de feuille synthèse a été conçu pour certaines banques. Finalement, la documentation d'accompagnement de chacune des banques de données sur disque optique est située près des postes de consultation. Les utilisateurs peuvent donc s'y référer en tout temps.

\section{Utilisation}

Les statistiques d'utilisation du SRIDO sont recueillies automatiquement par un programme maison rédigé en langage $C$ qui sert également d'interface-menu. II prend en note le statut de l'usager, la banque de données interrogée, l'heure du début et de la fin de l'interrogation.

L'utilisation du SRIDO montre une progression continue depuis son inauguration en juin 1990. Toutefois, nous assistons depuis la session d'hiver 1992 à une stabilisation de l'achalandage à près de 700 interrogations mensuelles. De ce nombre, approximativement $30 \%$ des usagers se prévalent du privilège de réservation. Plusieurs personnes interrogent donc directement les banques de données lorsque les postes de consultation sont libres.

Les étudiants des cycles supérieurs constituent la principale clientèle du SRIDO avec $39 \%$ des recherches (figure 1 ). Viennent ensuite les étudiants du baccalauréat et les employés de la bibliothèque avec chacun $20 \%$ des interrogations. L'emploi du SRIDO à plusieurs fins (sélection de la documentation, vérifications bibliographiques et recherches documentaires) explique son utilisation élevée par le personnel.

La figure 2 présente la répartition des recherches selon les différentes banques de données. Compendex Plus est de loin la banque la plus utilisée avec $47 \%$ des recherches, ce qui est compréhensible puisqu'elle couvre l'ingénierie dans son ensemble et qu'elle répond le mieux aux besoins des étudiants des cycles supérieurs, des professeurs/chercheurs et d'une partie du personnel de la bibliothèque.

Impacts

L'impact du SRIDO sur les autres services de la bibliothèque est difficilement quantifiable. Toutefois, une augmentation d'environ $5 \%$ du nombre de questions 
à la référence et des demandes de prêts entre bibliothèques a été enregistrée. Quant au nombre de recherches informatisées, celui-ci a diminué de près de $30 \%$.

Au niveau du personnel, des efforts soutenus sont placés tant dans l'élaboration d'outils documentaires que dans la formation elle-même. La gestion du SRIDO, c'est-à-dire les procédures d'utilisation, l'acquisition du matériel, la promotion, le développement de collection, la budgétisation, etc., exige également une somme importante de travail.

\section{Développements futurs}

Deux autres banques seront mises en service en décembre 1992. II s'agit de Automated Patent Searching (brevets américains) et de MOVE (automobiles et transport). D'autres banques sont également à l'étude actuellement: World Atlas, Languages of the World, El Page One et Standard Infodisk.
Nous prévoyons aussi mettre en place un réseau informatique afin d'assurer une meilleure diffusion des banques actuellement disponibles. De typologie à jeton circulant ("token ring»), ce réseau sera géré à l'aide des logiciels d'exploitation OS/2 et OS/2 Lan Server. Les tests sont en cours et la mise en service devrait avoir lieu à l'hiver 1993. II sera éventuellement relié au réseau de l'École. Parmi les autres projets, la rédaction d'une politique de développement de collection pour les ouvrages sur disque optique est en voie d'élaboration.

\section{Conclusion}

Les principaux aspects reliés à la mise en place du service de repérage de l'information sur disque optique à l'École Polytechnique ont été passés en revue. Globalement, l'arrivée du SRIDO a rehaussé l'image de la Bibliothèque au sein de la communauté polytechnicienne tout en favorisant l'utilisation des services et ressources existantes. Le personnel apprécie ce nouvel outil que sont les banques de données sur disque optique. Le SRIDO est maintenant un service important de la Bibliothèque et nous comptons sur sa popularité pour promouvoir d'autres services, telle la téléréférence.

En terminant, mentionnons également l'importance de déterminer préalablement les procédures d'utilisation du service et d'élaborer les principaux outils documentaires. Dans notre cas, cette étape fut très bénéfique puisqu'elle a favorisé une implantation harmonieuse du SRIDO. D'autre part, il est tout aussi important d'impliquer dès le départ toutes les sections concernées par un SRIDO. Par exemple, l'implication des systèmes informatisés est utile, voire nécessaire, à l'installation et la configuration des logiciels, à l'achat de matériel, etc. Nous l'avons réalisé un peu tardivement! C'est seulement au printemps 91 que les systèmes informatisés ont été officiellement chargés des aspects informatiques du SRIDO.

\section{La plus grande source d'informations sur la société québécoise!}

\section{La Bibliothèque québécoise vous propose}

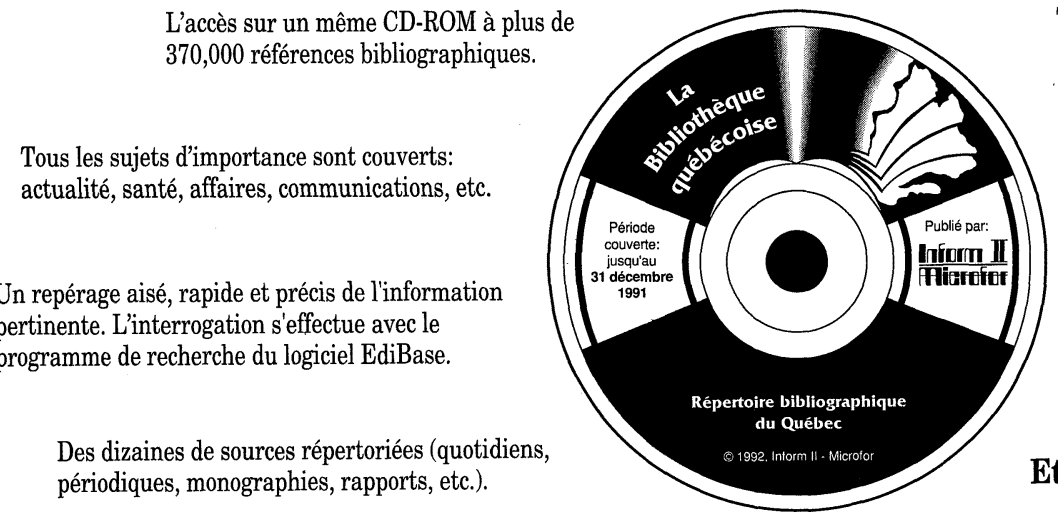

L'accès sur un même CD-ROM à plus de 370,000 références bibliographiques.

Tous les sujets d'importance sont couverts: actualité, santé, affaires, communications, etc.

Un repérage aisé, rapide et précis de l'information pertinente. L'interrogation s'effectue avec le programme de recherche du logiciel EdiBase.

Des dizaines de sources répertoriées (quotidiens, périodiques, monographies, rapports, etc.)
Tous les index produits par Inform II - Microfor: L'Index de l'actualité de 1982 à 1991, l'Index des affaires de 1988 à 1991, l'Index de la santé et des services sociaux de 1989 à 1991, ainsi que la base de données Médiadoq produite par le RQIC (1958 à 1991).

Un outil de travail bibliographique.

À partir des documents repérés, vous pouvez produire une variété de sorties imprimées (bibliographies, bulletins, index bibliographiques), ou transférer ces références dans vos propres bases de données.

Et plus encore à découvrir ! 